\title{
Metformin prevents metabolic side effects during systemic glucocorticoid treatment
}

\author{
Eleonora Seelig1, ${ }^{1,}$, Stefanie Meyer ${ }^{1, *}$, Katharina Timper ${ }^{1,2}$, Nicole Nigro1, \\ Martina Bally ${ }^{3}$, Ida Pernicova ${ }^{4}$, Philipp Schuetz ${ }^{3}$, Beat Müller ${ }^{3}$, Marta Korbonits ${ }^{4}$ \\ and Mirjam Christ-Crain'1
}

1Department of Endocrinology, Diabetology and Metabolism, University Hospital Basel, Basel, Switzerland, ${ }^{2}$ Max-Planck-Institute for Metabolism Research, Cologne, Germany, ${ }^{3}$ Division of Endocrinology, Diabetology and Metabolism, Medical University Clinic, Kantonsspital Aarau, Aarau, Switzerland, and ${ }^{4}$ Department of Endocrinology, William Harvey Research Institute, Barts and the London School of Medicine and Dentistry, Queen Mary University of London, London, UK *(E Seelig and S Meyer contributed equally to this work)

\author{
Correspondence \\ should be addressed \\ to M Christ-Crain \\ Email \\ mirjam.christ@usb.ch
}

\section{Abstract}

Objectives: Patients receiving glucocorticoid treatment are prone to develop metabolic complications. In preclinical studies, metformin prevented the development of the metabolic syndrome during glucocorticoid excess. We herein investigated the metabolic effect of metformin during glucocorticoid treatment in non-diabetic patients. Methods: In a double-blind, placebo-controlled trial, patients starting glucocorticoid treatment (prednisone, prednisolone or methylprednisolone) for four weeks were randomised to concomitantly receive metformin ( $850 \mathrm{mg}$ once daily for one week followed by $850 \mathrm{mg}$ twice daily for three weeks) or placebo. All patients underwent a standardised oral glucose tolerance test at baseline and after four weeks. The primary endpoint was change in the 2-h area under the curve (AUC) of glucose during the oral glucose tolerance test between baseline and four weeks. Results: 29 of 34 randomised non-diabetic patients completed the trial (17 metformin and 12 placebo). In patients allocated to placebo, median glucose 2-h AUC increased from baseline to four weeks (836 (IQR 770-966) to 1202 (1009-1271) $\mathrm{mmol} / \mathrm{L}$ per $\mathrm{min} ; P=0.01)$. In contrast, glucose levels remained similar to baseline in the metformin group (936 (869-1003) to 912 (825-1011) $\mathrm{mmol} / \mathrm{L}$ per $\min ; P=0.83$ ). This change within four weeks was different between both groups $(P=0.005)$. Glucocorticoid equivalent doses were similar in both groups (placebo: 980.0 (560.0-3259.8) mg/28 days; metformin: 683.0 (437.5-1970.5) mg/28 days; $P=0.26$ ).

Conclusions: In this first randomised controlled trial of metformin targeting metabolic complications in patients needing glucocorticoid therapy, we observed a beneficial effect of metformin on glycaemic control. Metformin thus seems to be a promising drug for preventing metabolic side effects during systemic glucocorticoid treatment.

\section{Introduction}

Up to $2.5 \%$ of the adult western population receive systemic glucocorticoid therapy, mostly for inflammatory conditions (1). Diabetes mellitus, dyslipidaemia, central obesity and hypertension are well-known and common side effects of glucocorticoid treatment $(2,3)$. Especially, diabetes mellitus is a recurring problem with a reported prevalence of up to $40 \%$ in patients receiving long-term glucocorticoid treatment $(4,5,6,7,8)$. Even if used as an antiemetic drug in cancer patients, glucocorticoids clearly increased the risk of diabetes mellitus (8). In contrast to other well-known side effects of glucocorticoids, such as gastric ulcer disease, no randomised controlled evidence exists that has investigated the potential therapeutics for the treatment of metabolic side effects of glucocorticoids.
() 2017 European Society of Endocrinology Printed in Great Britain
Published by Bioscientifica Ltd. 
Many of the changes seen in glucocorticoid excess, such as gluconeogenesis, correspond to metabolic steps regulated by adenosine monophosphate-activated protein kinase (AMPK) (9). AMPK is a key regulator of energy metabolism and mediator of several hormones affecting appetite and metabolism (10). Metformin, a widely used drug for prevention and treatment of diabetes mellitus type 2, exerts most of its beneficial effects on metabolism through the activation of $\operatorname{AMPK}(11,12)$. We have shown previously that glucocorticoid treatment changes AMPK activity in human adipocytes in vitro and reduced AMPK activity is seen in adipose tissue of patients with Cushing's syndrome $(13,14)$. Importantly, metformin reversed the effects of corticosteroids on AMPK in vitro both in primary hypothalamic cell culture as well as in adipocytes, suggesting that metformin and glucocorticoids influence the AMPK signalling pathway in opposite ways and that the metformin effect is able to override the cortisol effect $(13,15)$. In vivo studies showed that treatment with an AMPK activator prevented glucocorticoid-induced increase in glucose levels, hepatic glycogen production and hepatic steatosis in rats (16). Furthermore, metformin efficiently prevented the dexamethasone-induced deterioration of glucose metabolism in mice and horses $(17,18)$. These data suggest that metformin treatment could be beneficial in preventing metabolic complications in patients receiving long-term corticosteroid treatment.

In the first double-blind, randomised, placebocontrolled trial, we investigated the metabolic effects of metformin during glucocorticoid treatment in nondiabetic patients starting treatment with corticosteroids for at least 4 weeks.

\section{Materials and methods}

\section{Study design}

In this randomised, placebo-controlled, double-blind study, we included patients starting glucocorticoid treatment for at least 4 weeks. Participants were recruited at several departments at the University Hospital Basel and the Cantonal Hospital Aarau from August 2010 to March 2015. Patients were randomised in a 1:1 ratio to receive either metformin $850 \mathrm{mg}$ daily p.o. for one week followed by $850 \mathrm{mg}$ twice daily p.o. for another three weeks or identical placebo (Merck, Germany). The study was terminated after four weeks in all patients, also in cases where glucocorticoid treatment was continued. The study was registered at Clinicaltrials.gov NCT01187849.

\section{Patients}

Inclusion criterion was a newly initiated treatment with prednisone $\geq 7.5 \mathrm{mg}$ or an equivalent glucocorticoid for at least 4 weeks. Glucocorticoid tapering was determined by the treating physicians. Exclusion criteria were preexisting diabetes mellitus (according to the American Diabetes Association criteria); renal insufficiency (estimated glomerular filtration rate using the CKDEPI formula above $60 \mathrm{~mL} / \mathrm{min} / 1.73$ ); severe conditions affecting renal function (e.g. dehydration, fever and severe infection); severe conditions causing tissue hypoxia (e.g. acute cardiac or respiratory insufficiency); scheduled examination using intravascular contrast agent containing iodine; alcohol consumption of more than $40 \mathrm{~g} /$ day (male) or $20 \mathrm{~g} /$ day (female); known allergy to metformin; pregnancy or breast feeding and any condition compromising the ability of the subject to give written informed consent.

The study was approved by the ethical committees of the participating hospitals and Swissmedic and was conducted in accordance with the ethical guidelines of the Declaration of Helsinki. Written informed consent was obtained from all participating subjects before randomisation.

\section{Study assessment}

At baseline and after four weeks, a standardised 2-h $75 \mathrm{~g}$ oral glucose tolerance test was performed. After an overnight fast, baseline blood samples for fasting glucose, insulin, HbA1c, a full lipid profile and safety blood measurements were taken directly before ingestion of glucose. Additional blood samples for glucose were taken 30, 60, 90 and $120 \mathrm{~min}$ thereafter. Physical examination and urine analysis were performed, and doses of glucocorticoids were assessed at both visits. After one week, a telephone call took place to assess the compliance, adverse events and dosage of glucocorticoids. Three forms of glucocorticoids were prescribed: prednisone, prednisolone and methylprednisolone (Supplementary Table 1 , see section on supplementary data given at the end of this article). If needed, doses of glucocorticoids, e.g. methylprednisolone, were converted to equivalent doses of prednisone (19). Due to glucocorticoid tapering, cumulative glucocorticoid doses were calculated as follows: area under the curve was calculated using glucocorticoid doses at baseline, one and four weeks. The average daily prednisone dose was calculated as the area under the curve of the 28 study days. 
Plasma glucose and lipids were measured with enzymatic assays (cobas modular analyzer, Roche Diagnostics). Serum insulin and c-peptide were assessed using immune assays (IMMULITE 2000, Siemens). HbA1c was analysed in EDTA plasma with high-performance liquid chromatography (G8 HPLC Analyzer, Tosho Bioscience, San Francisco, CA, USA). Measurements of all blood parameters were taken in the routine central laboratory unit of the University Hospital Basel. The reported HOMA index was calculated according to Matthews et al. (20). Body impedance analysis (Body Impedance Analyzer Model BIA 101, Akern Srl, Florence, Italy) was performed to assess body composition and energy expenditure.

A randomisation list based on single sequence of random assignments was created by the Pharmaceutical Unit of the University Hospital Basel. Patients as well as study personnel were blinded to the medication allocation.

\section{Study end points}

The predefined primary endpoint was the change in the area under the concentration-time curve (AUC) for glucose during the $75 \mathrm{~g}$ oral glucose tolerance test between baseline and four weeks. Predefined secondary endpoints included change in fasting glucose levels, glycated haemoglobin levels (HbA1c), Homeostatis Model Assessment (HOMA) index, fasting lipid levels, body mass index, body composition and waist/hip ratio.

\section{Statistical analysis}

According to the protocol, the primary analysis followed the intention-to-treat principle, i.e. patients with complete follow-up were analysed in the groups to which they were randomised. Patients in the metformin group were expected to have unchanged 2-h glucose levels after ingestion of $75 \mathrm{~g}$ glucose, whereas patients in the placebo group would have an increase of approximately $25 \%$. Based on these assumptions, a sample size of 66 patients (33 per arm) was calculated to detect a significant difference between these distributions with a power of 90\% at the two-sided 5\% level. Discrete variables are expressed as counts (percentages) and continuous variables as median (interquartile range (IQR)). To compare changes across treatment groups the Mann-Whitney $U$ test was used for continuous data and the Fisher's exact test for categorical data. The Wilcoxon signed-rank test was used for comparisons within subjects. Incremental AUC for glucose values (during 120 min of a standardised oral glucose tolerance test) and glucocorticoid doses (during 28 days of study duration) was calculated using the trapezoid rule. To adjust for relevant covariates, linear regression analyses were employed. $P$ value $<0.05$ was defined as significant. Data were analysed using statistical software (Statistical Package for Social Sciences IBM SPSS, version 22). Figures were drawn using GraphPad Prism (GraphPad Software Inc.).

\section{Results}

\section{Baseline characteristics}

34 individuals were randomly assigned (1:1) to receive metformin $(n=20)$ or placebo $(n=14)$. In the metformin group, two patients withdrew from the study due to gastrointestinal symptoms and vertigo respectively; another patient was lost to follow-up after the baseline visit. In the placebo group, one patient did not receive glucocorticoids and one patient was lost to follow-up. A total of 17 subjects in the metformin group and 12 subjects in the placebo group completed the trial (Fig. 1). Patients in both treatment groups were well matched for baseline characteristics (Table 1). Baseline glucocorticoid doses were similar in both groups (metformin: 35.0 (11.3-50.0) mg/day; placebo: 30.0 (20.0-362.5) mg/day; $P=0.48)$. A comparison between patients completing the trial $(n=29)$ and patients dropping out $(n=5)$ showed no

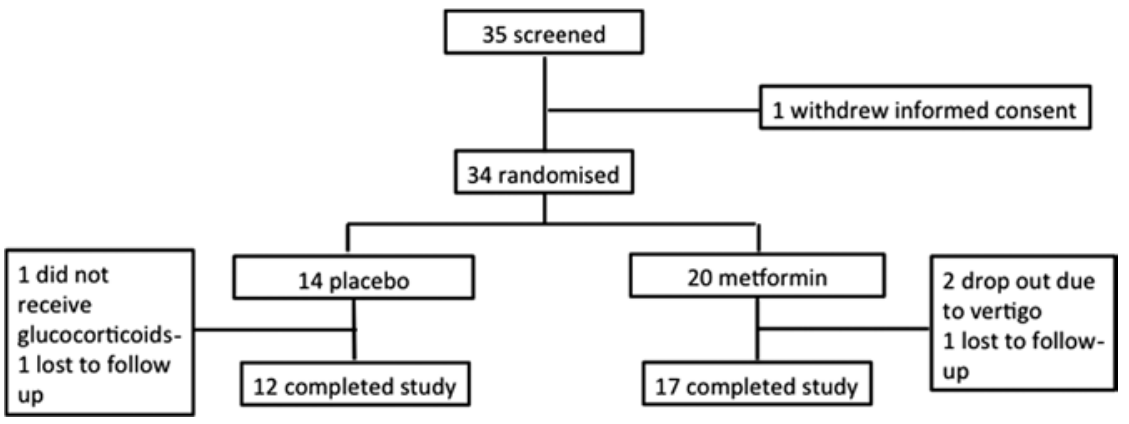

Figure 1

Enrolment of participants. 
Table 1 Baseline characteristics (including 5 patients with missing outcome variables); median values (IQR).

\begin{tabular}{l}
\hline \\
Male sex (\%) \\
Age (years) \\
BMI (kg/m²) \\
Waist/hip ratio \\
Systolic blood pressure $(\mathrm{mmHg})$ \\
Diastolic blood pressure $(\mathrm{mmHg})$ \\
HbA1c (\%) \\
HbA1c (mmol/mol) \\
Fasting glucose (mmol/L) \\
Fasting insulin (mIU/L) \\
HOMA index \\
Glucose $2 \mathrm{~h} \mathrm{AUC} \mathrm{(mmol/L} \mathrm{per} \mathrm{min)}$ \\
Triglycerides (mmol/L) \\
Total cholesterol (mmol/L) \\
HDL cholesterol (mmol/L) \\
LDL cholesterol (mmol/L) \\
Creatinine (umol/L) \\
Prednisone dosage (mg/day) \\
Basal metabolic rate (kcal) \\
Fat free mass (kg) \\
Fat mass (kg)
\end{tabular}

\begin{tabular}{c}
\hline Placebo $(n=14)$ \\
35.7 \\
$56.5(46.5-67.8)$ \\
$25.7(20.6-27.5)$ \\
$0.9(0.8-1.0)$ \\
$129(120-147)$ \\
$80(71-86)$ \\
$5.7(5.4-5.9)$ \\
$39.0(36.0-40.0)$ \\
$5.0(4.6-5.3)$ \\
$5.8(2.5-11.1)$ \\
$1.0(0.5-2.0)$ \\
$864.8(782.6-1012.1)$ \\
$1.1(0.9-1.2)$ \\
$4.8(4.4-5.2)$ \\
$1.4(1.0-1.7)$ \\
$2.9(2.6-3.1)$ \\
$67.0(60.8-75.5)$ \\
$30.0(20.0-362.5)$ \\
$1665(1423-1923)$ \\
$57.0(47.2-62.9)$ \\
$16.9(9.3-22.1)$
\end{tabular}

\begin{tabular}{ccc}
\hline Metformin $(n=20)$ & & $\begin{array}{c}P \\
\text { value }\end{array}$ \\
$58.0(35.0$ & & 0.08 \\
$24.2(21.6-28.6)$ & 0.69 \\
$1.0(0.9-1.0)$ & 0.69 \\
$132(116-139)$ & 0.24 \\
$75(70-80)$ & 0.96 \\
$5.4(5.3-5.8)$ & 0.26 \\
$36.0(34.0-40.0)$ & 0.32 \\
$4.8(4.6-5.3)$ & 0.32 \\
$8.6(4.3-14.8)$ & 0.77 \\
$1.9(1.0-3.4)$ & 0.29 \\
$937.5(872.3-991.1)$ & 0.18 \\
$1.3(0.9-1.7)$ & 0.34 \\
$4.8(4.3-5.6)$ & 0.32 \\
$1.2(1.0-1.4)$ & 0.64 \\
$3.1(2.5-3.8)$ & 0.48 \\
$79.0(59.8-87.3)$ & 0.27 \\
$35.0(11.3-50.0)$ & 0.27 \\
$1730(1593-1823)$ & 0.48 \\
$57.9(50.6-63.3)$ & 0.60 \\
$14.6(8.5-21.2)$ & 0.70 \\
& 0.77 \\
\hline
\end{tabular}

difference in baseline criteria except for glucocorticoid doses (complete: 40.0 (20.0-95.0) mg/day; drop out: 12.5 (10.0-26.3) mg/day, $P=0.03$ ) (Supplementary Table 2). AUC prednisone doses in patients completing the trial remained similar in both groups throughout the study (metformin: 683.0 (437.5-1970.5) $\mathrm{mg} / 28$ days; placebo: $980.0(560.0-3259.8) \mathrm{mg} / 28$ days, $P=0.26$ ). Indications for glucocorticoid treatment are presented in Table 2 . Concomitant medication with potential effect on glucose

Table 2 Indications for glucocorticoid treatment (including 5 patients with missing outcome variables).

\begin{tabular}{|c|c|c|}
\hline Diagnosis & $\begin{array}{c}\text { Placebo } \\
(n=14)\end{array}$ & $\begin{array}{c}\text { Metformin } \\
\quad(n=20)\end{array}$ \\
\hline Arthritis & 2 & 2 \\
\hline Vasculitis & 1 & 3 \\
\hline Polymyalgia rheumatica & 1 & 2 \\
\hline Eosinophilic fasciitis & 1 & \\
\hline Lupus erythematodes & 1 & \\
\hline Sarcoidosis & & 2 \\
\hline Sclerosing lymphadenopathy & 1 & \\
\hline Cutaneous sclerosis & & 1 \\
\hline Morbus-Wegener & 1 & \\
\hline Alopecia areata & & 1 \\
\hline Pemphigus & 2 & 1 \\
\hline Eczema & & 1 \\
\hline Metastatic prostate carcinoma & & 1 \\
\hline Astrocytoma & 1 & \\
\hline Organizing pneumonia & & 1 \\
\hline Allergic bronchopulmonary aspergillosis & & 1 \\
\hline Myasthenia gravis & & 1 \\
\hline Endocrine orbitopathy & 3 & 2 \\
\hline Scleritis & & 1 \\
\hline
\end{tabular}

and/or lipid metabolism is listed in Supplementary Table 3. Due to slow study recruitment and time of expiry of study drug, the study had to be prematurely terminated. This led to fewer study participants than intended and to an unbalanced randomisation.

\section{Effect of metformin on glycaemia}

Two-hour AUC glucose remained similar from baseline to four weeks in the metformin group $(P=0.83)$, whereas it was increasing in the placebo group $(P=0.01$; Fig. $2 \mathrm{~A}, \mathrm{~B}$ and $\mathrm{C}$ ). Accordingly, the primary endpoint of 2-h AUC glucose change within four weeks was different between both groups ( $P=0.005$; Table 3; Fig. $2 \mathrm{D})$. After adjustment for gender, cumulative glucocorticoid dose and HbA1c, treatment group remained strongly associated with 2-h AUC glucose (adjustment for gender: treatment group $P=0.006, R^{2}=0.32$; adjustment for glucocorticoid dose: treatment group $P=0.003, R^{2}=0.33$; adjustment for HbA1c: treatment group $P=0.002, R^{2}=0.38$ ). Among the secondary endpoints, the change in fasting glucose, fasting insulin and HOMA index were different between the two groups ( $P=0.01, P=0.003$ and $P=0.035$ respectively; Fig. $3 \mathrm{~A}, \mathrm{~B}$, $\mathrm{C}, \mathrm{D}, \mathrm{E}$ and $\mathrm{F}$ ). We observed no change in HbA1c in the treatment and placebo groups during the study period $(P=0.64$; Fig. $3 \mathrm{G}$ and $\mathrm{H})$.

Furthermore, we aimed to differentiate between responders and non-responders to metformin. As the 2-h AUC glucose increase in the placebo group was 40.3 
A
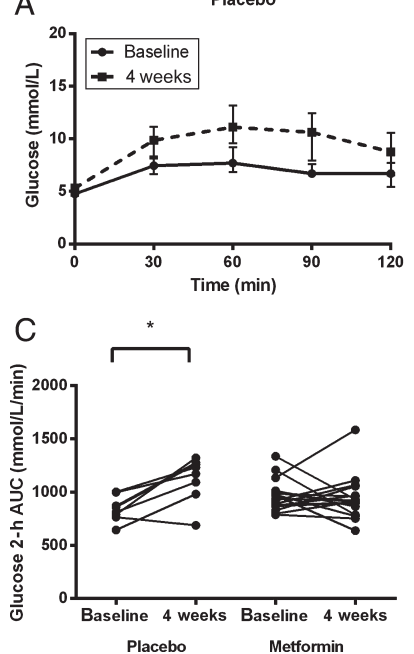

Placebo

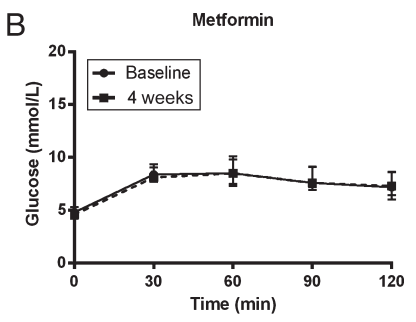

D

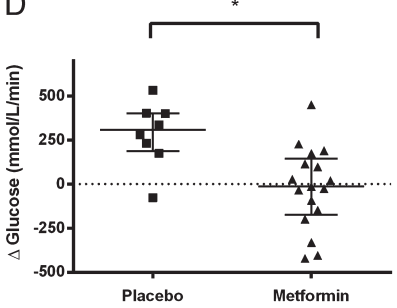

Figure 2

Change in glucose during oral glucose tolerance test.

(A) Plasma glucose values during oral glucose tolerance test at baseline and after four weeks in placebo-treated patients.

(B) Glucose values during oral glucose tolerance test at baseline and after four weeks in patients treated with metformin. (C) 2-h AUC glucose in both study groups at baseline and after 4 weeks. (D) Differences in 2-h AUC glucose between baseline and four weeks in each study group.

Data represent median values error bars indicate interquartile ranges. ${ }^{*} P$ value $<0.05$.

(18.9-51.0)\%, we allocated patients in the metformin group with an increase below 20\% to responders, and patients with an increase equal and above $20 \%$ to nonresponders. This resulted in three patients, who were classified as non-responders. Their baseline characteristics are listed in Supplementary Table 4.

\section{Effect of metformin on lipids}

Fasting triglyceride levels did not change during the trial, and there was no difference between groups $(P=0.30)$. Total cholesterol levels increased only in the placebo group $(P=0.02)$ while remaining stable in the metformin group $(P=0.10)$. No difference in cholesterol between groups was observed $(P=0.15)$. HDL levels increased in both groups compared to baseline (metformin: $P<0.0001$; placebo: $P=0.003)$. The HDL increase over the four weeks was more pronounced in the metformin group $(P=0.04)$. LDL levels did not change during the trial, and there was no difference between groups $(P=0.71$; Table 3$)$.

\section{Effect of metformin on body composition and energy expenditure}

We identified no change in BMI, waist-to-hip ratio, basal metabolic rate, fat free mass and fat mass during the study period; there was no difference across treatment groups (Table 3).

\section{Adverse events}

Gastrointestinal symptoms were present in $20.0 \%$ of patients in the metformin and in $21.4 \%$ of patients in the placebo group (Supplementary Table 5). All gastrointestinal symptoms were either mild or moderate. There was no difference between groups $(P=0.99)$. In the metformin group, one subject discontinued the study due to gastrointestinal symptoms, and another patient discontinued due to vertigo. One subject in the metformin group was hospitalised for further evaluation of the underlying disease (vasculitis) after study inclusion. The hospitalisation was rated as serious adverse event unrelated to the study drug.

\section{Discussion}

In this trial, with non-diabetic patients receiving systemic glucocorticoids, we demonstrate for the first time that preventive metformin treatment is superior to placebo with respect to glycaemic control as indicated by 2-h glucose AUC, HOMA index, fasting glucose and fasting insulin. This effect was consistent after adjustment for gender, cumulative glucocorticoid dose and HbA1c. Although HDL cholesterol levels increased in both groups during GC treatment, we did not observe a change in triglycerides, LDL, body weight or body composition.

Despite the very frequent use of glucocorticoids and the well-known detrimental impact on glucose metabolism, hardly any randomised controlled trials have investigated the prevention of glucocorticoid-induced diabetes (21, $22,23,24)$. In one of these trials, troglitazone prevented deterioration of glucose metabolism during glucocorticoid treatment, whereas pioglitazone and metformin had no effect (25). Noteworthy, troglitazone can no longer be used as it was withdrawn from the market. Compared to our study, duration of metformin and steroid treatment was very short and metformin dose was low. Two other randomised controlled trials targeting the GLP-1 pathway produced heterogeneous results $(26,27)$. Importantly, all three studies were performed in individuals without 
Table 3 Primary and secondary endpoints; median values (IQR); for each parameter, change from baseline was compared between groups (metformin vs placebo) using the Mann-Whitney $U$ test and within-groups using the Wilcoxon signed-rank test.

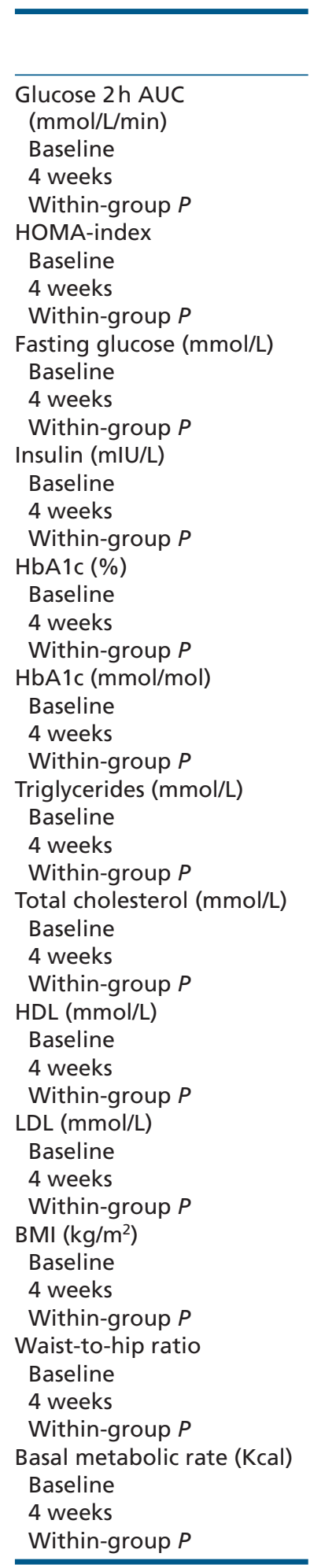

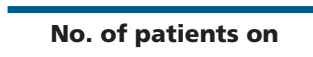
metformin vs placebo 17 vs 8

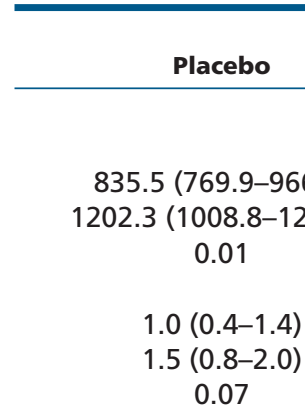

17 vs 11

17 vs 9

$$
\begin{gathered}
1.0(0.4-1.4) \\
1.5(0.8-2.0) \\
0.07
\end{gathered}
$$

$$
\begin{gathered}
4.8(4.4-5.3) \\
5.3(4.5-5.6) \\
0.07
\end{gathered}
$$

17 vs 10

$$
\begin{gathered}
5.4(2.3-8.3) \\
6.8(4.0-13.4) \\
0.07
\end{gathered}
$$

16 vs 12

$$
\begin{gathered}
5.7(5.3-5.9) \\
5.8(5.3-5.9) \\
0.19
\end{gathered}
$$

16 vs 12

$$
\begin{gathered}
39.0(34.0-41.0) \\
40.0(34.0-41.0) \\
0.19
\end{gathered}
$$

17 vs 11

$$
\begin{gathered}
1.1(0.8-1.1) \\
1.2(0.9-1.3) \\
0.17
\end{gathered}
$$

17 vs 11

$$
\begin{gathered}
4.8(4.5-5.1) \\
5.6(4.8-6.9) \\
0.02
\end{gathered}
$$

17 vs 11

17 vs 11

$$
\begin{gathered}
1.5(1.0-1.6) \\
2.0(1.7-2.8) \\
0.003
\end{gathered}
$$

$$
\begin{gathered}
2.9(2.6-3.1) \\
3.0(2.7-3.4) \\
0.53
\end{gathered}
$$

17 vs 12

$$
\begin{gathered}
25.7(21.9-26.4) \\
25.5(21.4-27.4) \\
0.72
\end{gathered}
$$

16 vs 9

$$
\begin{gathered}
0.9(0.8-1.0) \\
1.0(0.9-1.0) \\
0.17
\end{gathered}
$$

14 vs 10

$$
\begin{gathered}
1665(1523-1888) \\
1620(1418-1952) \\
0.65
\end{gathered}
$$

Between group $P$ 0.005

$936.0(869.3-1002.8)$

912.0 (825.0-1011.0) 0.83

$$
\begin{gathered}
2.2(1.0-3.6) \\
1.1(0.6-2.7) \\
0.04
\end{gathered}
$$

$$
\begin{gathered}
4.8(4.6-5.3) \\
4.6(4.2-5.0) \\
0.04
\end{gathered}
$$

$$
\begin{gathered}
9.3(4.5-15.6) \\
5.7(3.3-13.4) \\
0.06
\end{gathered}
$$

$$
\begin{gathered}
5.4(5.3-6.0) \\
5.5(5.3-6.0) \\
0.48
\end{gathered}
$$

$$
\begin{gathered}
36.0(34.0-42.0) \\
37.0(34.0-42.0) \\
0.48
\end{gathered}
$$

0.003

0.64

0.64

$1.3(0.9-1.6)$

$1.2(1.0-1.4)$

$$
0.65
$$

$$
\begin{gathered}
4.8(4.2-5.7) \\
5.4(4.6-6.4) \\
0.10
\end{gathered}
$$

$$
\begin{gathered}
1.3(1.1-1.5) \\
1.7(1.3-1.9) \\
<0.0001
\end{gathered}
$$

$$
\begin{gathered}
3.0(2.3-3.9) \\
3.0(2.5-3.8) \\
0.83
\end{gathered}
$$

$$
\begin{gathered}
23.7(20.9-28.7) \\
23.6(21.1-28.7) \\
0.26
\end{gathered}
$$

$$
\begin{gathered}
1.0(0.9-1.0) \\
1.0(0.9-1.0) \\
0.93
\end{gathered}
$$

$$
\begin{gathered}
1730(1550-1835) \\
1745(1513-1820) \\
0.55
\end{gathered}
$$

0.95

(Continued) 
Table 3 Continued.

\begin{tabular}{|c|c|c|c|c|}
\hline & $\begin{array}{l}\text { No. of patients on } \\
\text { metformin vs placebo }\end{array}$ & Placebo & Metformin & $\begin{array}{l}\text { Between } \\
\text { group } P\end{array}$ \\
\hline Fat free mass $(\mathrm{kg})$ & 14 vs 10 & & & 0.38 \\
\hline Baseline & & $57.0(47.5-62.3)$ & $58.3(52.9-63.9)$ & \\
\hline 4 weeks & & $54.7(41.6-63.2)$ & $57.7(51.5-64.7)$ & \\
\hline Within-group $P$ & & 0.37 & 0.95 & \\
\hline Fat mass $(\mathrm{kg})$ & 14 vs 10 & & & 0.98 \\
\hline Baseline & & $16.9(10.4-21.3)$ & $14.6(9.8-22.3)$ & \\
\hline 4 weeks & & $19.2(12.1-22.8)$ & $17.1(9.5-22.9)$ & \\
\hline Within-group $P$ & & 0.59 & 0.35 & \\
\hline $\begin{array}{l}\text { AUC prednisone dosage } \\
\text { (mg/28 days) }^{a}\end{array}$ & 17 vs 12 & $980.0(560.0-3259.8)$ & $683.0(437.5-1970.5)$ & 0.26 \\
\hline
\end{tabular}

aPrednisone dosage was calculated as area under the curve using glucocorticoid doses at baseline, one and four weeks.

inflammatory disease, thus not representing the patients in need of glucocorticoid treatment. As inflammation is a known mediator of insulin resistance, it is important to investigate the potential benefits of metformin in an appropriate study population (28). Therefore, more convincing strategies to prevent metabolic side effects of glucocorticoid treatment in patients indeed suffering from inflammatory diseases are needed.

From a pathophysiological point of view, metformin is an attractive preventive treatment strategy in patients receiving corticosteroids. Metformin's mode of function has been extensively discussed, and several mechanisms such as inhibition of glycerol phosphate dehydrogenase, enhanced action of glucagon-like-peptide 1 or antagonism of glucagon have been proposed $(29,30,31,32)$. Overall, the activation of AMPK seems to play an important role $(11,12,33,34)$. AMPK is generally considered to be a master regulator of energy metabolism, sensing energy depletion and activating energy-generating pathways (10). Glucocorticoids have been shown to inhibit AMPK activity and, importantly, metformin was able to reverse this inhibitory effect of glucocorticoids on AMPK in vitro and in animal studies $(13,14,16)$.

In accordance with these experimental data, our study showed that metformin favourably influences several side effects of glucocorticoid therapy. We found that metformin prevented an increase of 2 -h glucose AUC indicating preservation of glucose tolerance. The HOMA index, a marker of insulin resistance, clearly improved in the metformin group, whereas deterioration was observed in the placebo group. Fasting glucose levels decreased in the metformin group, whereas increased in the placebo group during the study period. Moreover, change in fasting insulin was different between groups. Still, we could not identify a difference in HbA1c. However, our study was conducted over four weeks while HbA1c reflects average blood glucose over the previous 8-12 weeks (35).
Therefore, we speculate that a longer study duration could show a beneficial effect on HbA1c.

Compared to glucose metabolism, the role of glucocorticoids in lipid metabolism is more controversial. Patients with endogenous overproduction of glucocorticoids are prone to develop dyslipidaemia (2). Similarly, glucocorticoid administration has been associated with the deterioration of lipid metabolism (36). Interestingly, in a large observational study, glucocorticoids were associated with higher HDL levels and glucocorticoid treatment was shown to normalise HDL levels in rheumatoid arthritis $(37,38,39)$. This positive effect of glucocorticoids may be due to the reduction of the inflammatory burden rather than a direct impact on lipid metabolism.

Although the role of glucocorticoids on lipids remains unclear, metformin presumably has a beneficial effect by decreasing triglycerides and LDL cholesterol while increasing $\mathrm{HDL}$ cholesterol independent of glucose metabolism $(40,41,42)$. In our trial, we did not observe a change in triglycerides or LDL; however, HDL cholesterol levels increased in both study groups. This finding may be due to a direct effect of glucocorticoids or rather an indirect effect of lowering the inflammatory status.

Central obesity is another characteristic feature of chronic high-dose glucocorticoid exposure $(43,44)$. In the Diabetes Prevention Program Study, metformin reduced body weight for around $2 \mathrm{~kg}$ during a 2 -year study period in diabetic patients (45). Thus, metformin exerts opposite effects to glucocorticoids regarding weight.

In our trial, four weeks of glucocorticoid treatment did not result in change of body composition or waist/hip ratio in either study groups. Consequently, no effect of metformin could be observed. Possibly, the study duration was too short and the sample size was too small; longer treatment duration with corticosteroids and metformin or placebo respectively, may provide different results. 
A

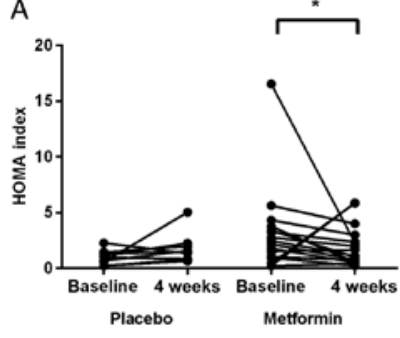

C

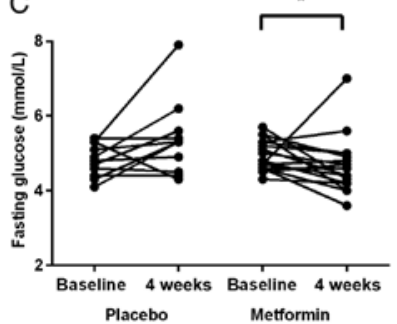

E

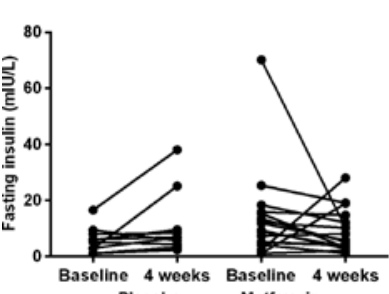

Placebo

Mettormin

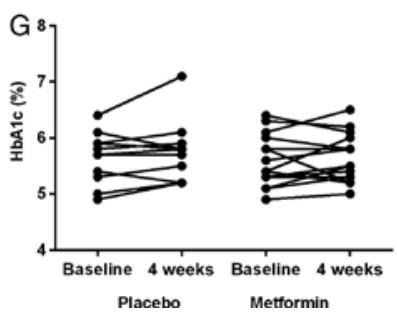

Placebo
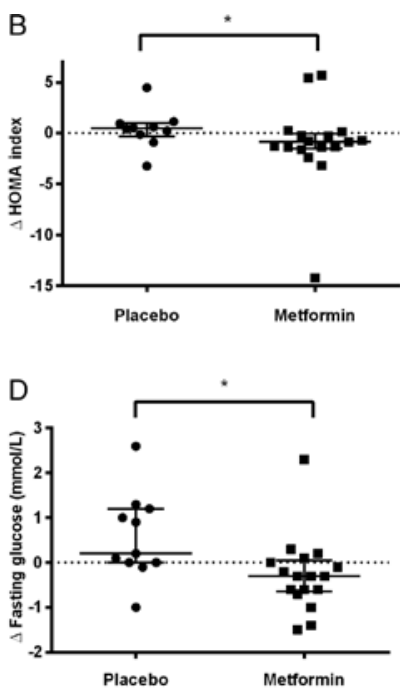

$\mathrm{F}$
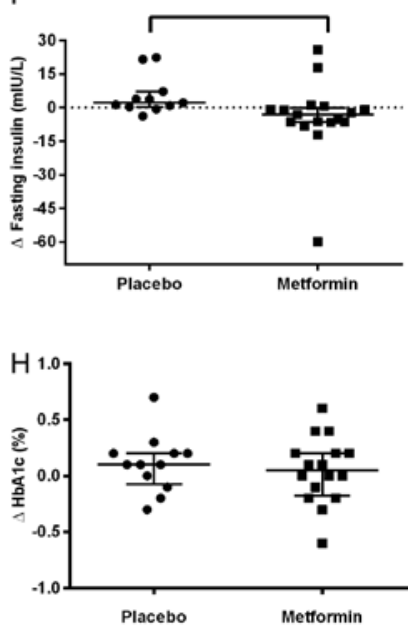

Figure 3

Change in HOMA index, fasting glucose, fasting insulin and HbA1c. (A) HOMA index at baseline and after four weeks for both study groups. (B) Differences in HOMA index between baseline and four weeks in each study group. (C) Fasting glucose at baseline and after four weeks in each study group. (D) Differences in fasting glucose between baseline and four weeks in each study group. (E) Fasting insulin at baseline and after four weeks in each study group. (F) Differences in fasting insulin at baseline and after four weeks in each study group.

(G) HbA1c at baseline and after four weeks in each study group. $(\mathrm{H})$ Differences in between baseline and four weeks in each study group. Data represent median values, error bars indicate interquartile ranges. ${ }^{*} P$ value $<0.05$.

Gastrointestinal adverse events occurred in similar number in both treatment groups. Several other studies found metformin to be safe and well tolerated (46).

Our study has some limitations. First, the study was prematurely terminated, which led to a rather small sample size. This was due to a combination of slow and difficult recruitment and time to expiry of the trial drug. Nevertheless, due to higher than expected effect of metformin, the sample size was sufficient to demonstrate a significant effect on the primary and several secondary endpoints. As we show a highly significant result, lack of statistical power is not an issue. Second, more and predominantly male patients were in the metformin group. Third, causes of glucocorticoid administration were very variable, and the study design did not allow stratification of diseases. Although overall glucocorticoid doses were not different between groups, some participants in the placebo group received the highest doses. Importantly, variability of indications and administration of glucocorticoid treatment mirror real life practice and make the results more generalisable. Fourth, baseline HbA1c was slightly higher in the placebo group, potentially putting these patients at higher risk for development of diabetes. Importantly, the difference in $\mathrm{HbA1c}$ was not significant between groups, and the primary endpoint remained highly significant after adjustment for HbA1c.

Our results indicate that metformin prevents deterioration of glucose metabolism if treatment is timed with the initiation of glucocorticoids. This study provides the basis for metformin as a preventive treatment in patients newly receiving glucocorticoid therapy. Further studies are needed to test if occurrence of glucocorticoidinduced diabetes can be reduced and if metformin has similar beneficial effects in patients with continuous glucocorticoid treatment. As our patient number was too small to identify unique characteristics distinguishing responders from non-responders, this remains to be investigated in future studies.

In summary, this is the first randomised controlled trial showing that metformin has a beneficial preventive effect on glycaemic control in non-diabetic patients receiving systemic glucocorticoid therapy.

Supplementary data

This is linked to the online version of the paper at http://dx.doi.org/ 10.1530/EJE-16-0653.

Declaration of interest

The authors declare that there is no conflict of interest that could be perceived as prejudicing the impartiality of the research reported. 
Funding

This work was supported by the Swiss National Research Foundation

\section{Author contribution statement}

M C-C designed the study. E S, S M, K T, N N and M B conducted the experiments. E S analysed the data. E S, S M and M C-C wrote the manuscript. K T, I P, P S, B M and M K reviewed and edited the manuscript. $\mathrm{M} \mathrm{C-C}$ is the guarantor of the study and, as such, takes responsibility for the contents of this article.

\section{Acknowledgement}

The authors are grateful for Merck, Germany to provide study drug and matching placebo; Dr Blerina Kola for her contribution in the initial planning of the study; Prof. Matthias Briel for statistical support; Dr Thomas Daikeler, Dr Peter Haeusermann, Dr Christof Rottenburger and Prof Viviane Hess for their support regarding recruitment; Susanne Ruesch and Cemile Bathelt for their support in conducting the trial. They especially thank Marc Donath for supporting the study.

\section{References}

1 van Staa TP, Leufkens HG, Abenhaim L, Begaud B, Zhang B \& Cooper C. Use of oral corticosteroids in the United Kingdom. QJM 200093 105-111. (doi:10.1093/qjmed/93.2.105)

2 Arnaldi G, Angeli A, Atkinson AB, Bertagna X, Cavagnini F, Chrousos GP, Fava GA, Findling JW, Gaillard RC, Grossman AB et al. Diagnosis and complications of Cushing's syndrome: a consensus statement. Journal of Clinical Endocrinology and Metabolism $2003 \mathbf{8 8}$ 5593-5602. (doi:10.1210/jc.2003-030871)

3 van Raalte DH, Ouwens DM \& Diamant M. Novel insights into glucocorticoid-mediated diabetogenic effects: towards expansion of therapeutic options? European Journal of Clinical Investigation 200939 81-93. (doi:10.1111/j.1365-2362.2008.02067.x)

4 Gulliford MC, Charlton J \& Latinovic R. Risk of diabetes associated with prescribed glucocorticoids in a large population. Diabetes Care 200629 2728-2729. (doi:10.2337/dc06-1499)

5 Clore JN \& Thurby-Hay L. Glucocorticoid-induced hyperglycemia. Endocrine Practice 200915 469-474. (doi:10.4158/EP08331.RAR)

6 Hoes JN, van der Goes MC, van Raalte DH, van der Zijl NJ, den Uyl D, Lems WF, Lafeber FP, Jacobs JW, Welsing PM, Diamant M et al. Glucose tolerance, insulin sensitivity and beta-cell function in patients with rheumatoid arthritis treated with or without low-tomedium dose glucocorticoids. Annals of the Rheumatic Diseases 2011 70 1887-1894. (doi:10.1136/ard.2011.151464)

7 Uzu T, Harada T, Sakaguchi M, Kanasaki M, Isshiki K, Araki S, Sugiomoto T, Koya D, Haneda M, Kashiwagi A et al. Glucocorticoidinduced diabetes mellitus: prevalence and risk factors in primary renal diseases. Nephron Clinical Practice 2007105 c54-c57. (doi:10.1159/000097598)

8 Jeong Y, Han HS, Lee HD, Yang J, Jeong J, Choi MK, Kwon J, Jeon HJ, $\mathrm{Oh} \mathrm{TK}$, Lee $\mathrm{KH}$ et al. A pilot study evaluating steroid-induced diabetes after antiemetic dexamethasone therapy in chemotherapy-treated cancer patients. Cancer Research and Treatment 201648 1429-1437. (doi:10.4143/crt.2015.464)

9 Pasieka AM \& Rafacho A. Impact of glucocorticoid excess on glucose tolerance: clinical and preclinical evidence. Metabolites $2016 \mathbf{6} 24$ (doi:10.3390/metabo6030024)

10 Kahn BB, Alquier T, Carling D \& Hardie DG. AMP-activated protein kinase: ancient energy gauge provides clues to modern understanding of metabolism. Cell Metabolism 20051 15-25. (doi:10.1016/j. cmet.2004.12.003)
11 Shaw RJ, Lamia KA, Vasquez D, Koo SH, Bardeesy N, Depinho RA, Montminy $\mathrm{M} \&$ \& Cantley LC. The kinase LKB1 mediates glucose homeostasis in liver and therapeutic effects of metformin. Science 2005310 1642-1646. (doi:10.1126/science.1120781)

12 Zhou G, Myers R, Li Y, Chen Y, Shen X, Fenyk-Melody J, Wu M, Ventre J, Doebber T, Fujii N et al. Role of AMP-activated protein kinase in mechanism of metformin action. Journal of Clinical Investigation 2001108 1167-1174. (doi:10.1172/JCI13505)

13 Christ-Crain M, Kola B, Lolli F, Fekete C, Seboek D, Wittmann G, Feltrin D, Igreja SC, Ajodha S, Harvey-White J et al. AMP-activated protein kinase mediates glucocorticoid-induced metabolic changes: a novel mechanism in Cushing's syndrome. FASEB Journal 200822 1672-1683. (doi:10.1096/fj.07-094144)

14 Kola B, Christ-Crain M, Lolli F, Arnaldi G, Giacchetti G, Boscaro M, Grossman AB \& Korbonits M. Changes in adenosine 5'-monophosphate-activated protein kinase as a mechanism of visceral obesity in Cushing's syndrome. Journal of Clinical Endocrinology and Metabolism 200893 4969-4973. (doi:10.1210/ jc. 2008-1297)

15 Foretz M, Guigas B, Bertrand L, Pollak M \& Viollet B. Metformin: from mechanisms of action to therapies. Cell Metabolism 201420 953-966. (doi:10.1016/j.cmet.2014.09.018)

16 Nader N, Ng SS, Lambrou GI, Pervanidou P, Wang Y, Chrousos GP $\&$ Kino T. AMPK regulates metabolic actions of glucocorticoids by phosphorylating the glucocorticoid receptor through p38 MAPK. Molecular Endocrinology 201024 1748-1764. (doi:10.1210/ me.2010-0192)

17 Rendle DI, Rutledge F, Hughes KJ, Heller J \& Durham AE. Effects of metformin hydrochloride on blood glucose and insulin responses to oral dextrose in horses. Equine Veterinary Journal 201345 751-754. (doi:10.1111/evj.12068)

18 Thomas CR, Turner SL, Jefferson WH \& Bailey CJ. Prevention of dexamethasone-induced insulin resistance by metformin. Biochemical Pharmacology 199856 1145-1150. (doi:10.1016/S00062952(98)00151-8)

19 Meikle AW \& Tyler FH. Potency and duration of action of glucocorticoids. Effects of hydrocortisone, prednisone and dexamethasone on human pituitary-adrenal function. American Journal of Medicine 197763 200-207. (doi:10.1016/00029343(77)90233-9)

20 Matthews DR, Hosker JP, Rudenski AS, Naylor BA, Treacher DF \& Turner RC. Homeostasis model assessment: insulin resistance and beta-cell function from fasting plasma glucose and insulin concentrations in man. Diabetologia 198528 412-419. (doi:10.1007/ BF00280883)

21 van Raalte DH, Diamant M, Ouwens DM, Ijzerman RG, Linssen MM Guigas B, Eringa EC \& Serne EH. Glucocorticoid treatment impairs microvascular function in healthy men in association with its adverse effects on glucose metabolism and blood pressure: a randomised controlled trial. Diabetologia 201356 2383-2391. (doi:10.1007/ s00125-013-3016-8)

22 van Raalte DH, Nofrate V, Bunck MC, van Iersel T, Elassaiss Schaap J, Nassander UK, Heine RJ, Mari A, Dokter WH \& Diamant M. Acute and 2-week exposure to prednisolone impair different aspects of beta-cell function in healthy men. European Journal of Endocrinology 2010162 729-735. (doi:10.1530/EJE-09-1034)

23 Rafacho A, Ortsater H, Nadal A \& Quesada I. Glucocorticoid treatment and endocrine pancreas function: implications for glucose homeostasis, insulin resistance and diabetes. Journal of Endocrinology 2014223 R49-R62. (doi:10.1530/JOE-14-0373)

24 Schacke H, Docke WD \& Asadullah K. Mechanisms involved in the side effects of glucocorticoids. Pharmacology \& Therapeutics 200296 23-43. (doi:10.1016/s0163-7258(02)00297-8)

25 Morita H, Oki Y, Ito T, Ohishi H, Suzuki S \& Nakamura H. Administration of troglitazone, but not pioglitazone, reduces insulin resistance caused by short-term dexamethasone (DXM) treatment by 
accelerating the metabolism of DXM. Diabetes Care 200124 788-789. (doi:10.2337/diacare.24.4.788)

26 van Raalte DH, van Genugten RE, Linssen MM, Ouwens DM \& Diamant M. Glucagon-like peptide-1 receptor agonist treatment prevents glucocorticoid-induced glucose intolerance and islet-cell dysfunction in humans. Diabetes Care 201134 412-417. (doi:10.2337/ dc10-1677)

27 van Genugten RE, van Raalte DH, Muskiet MH, Heymans MW, Pouwels PJ, Ouwens DM, Mari A \& Diamant M. Does dipeptidyl peptidase-4 inhibition prevent the diabetogenic effects of glucocorticoids in men with the metabolic syndrome? A randomized controlled trial. European Journal of Endocrinology 2014170 429-439. (doi:10.1530/EJE-13-0610)

28 Wellen KE \& Hotamisligil GS. Inflammation, stress, and diabetes. Journal of Clinical Investigation 2005115 1111-1119. (doi:10.1172/ JCI200525102)

29 Pernicova I \& Korbonits M. Metformin - mode of action and clinical implications for diabetes and cancer. Nature Reviews Endocrinology 201410 143-156. (doi:10.1038/nrendo.2013.256)

30 Madiraju AK, Erion DM, Rahimi Y, Zhang XM, Braddock DT, Albright RA, Prigaro BJ, Wood JL, Bhanot S, MacDonald MJ et al. Metformin suppresses gluconeogenesis by inhibiting mitochondrial glycerophosphate dehydrogenase. Nature 2014510 542-546. (doi:10.1038/nature13270)

31 Maida A, Lamont BJ, Cao X \& Drucker DJ. Metformin regulates the incretin receptor axis via a pathway dependent on peroxisome proliferator-activated receptor-alpha in mice. Diabetologia 201154 339-349. (doi:10.1007/s00125-010-1937-z)

32 Miller RA, Chu Q, Xie J, Foretz M, Viollet B \& Birnbaum MJ. Biguanides suppress hepatic glucagon signalling by decreasing production of cyclic AMP. Nature 2013494 256-260. (doi:10.1038/ nature11808)

33 He L, Sabet A, Djedjos S, Miller R, Sun X, Hussain MA, Radovick S $\&$ Wondisford FE. Metformin and insulin suppress hepatic gluconeogenesis through phosphorylation of CREB binding protein. Cell 2009137 635-646. (doi:10.1016/j.cell.2009.03.016)

34 Cool B, Zinker B, Chiou W, Kifle L, Cao N, Perham M, Dickinson R, Adler A, Gagne G, Iyengar R et al. Identification and characterization of a small molecule AMPK activator that treats key components of type 2 diabetes and the metabolic syndrome. Cell Metabolism 20063 403-416. (doi:10.1016/j.cmet.2006.05.005)

35 Nathan DM, Kuenen J, Borg R, Zheng H, Schoenfeld D, Heine RJ \& Group Ac-DAGS. Translating the A1C assay into estimated average glucose values. Diabetes Care 200831 1473-1478. (doi:10.2337/ dc08-0545)

36 Filipsson H, Monson JP, Koltowska-Haggstrom M, Mattsson A \& Johannsson G. The impact of glucocorticoid replacement regimens on metabolic outcome and comorbidity in hypopituitary patients. Journal of Clinical Endocrinology and Metabolism 200691 3954-3961. (doi:10.1210/jc.2006-0524)

37 Choi HK \& Seeger JD. Glucocorticoid use and serum lipid levels in US adults: the Third National Health and Nutrition Examination Survey. Arthritis \& Rheumatology 200553 528-535. (doi:10.1002/ art.21329)

38 Boers M, Nurmohamed MT, Doelman CJ, Lard LR, Verhoeven AC, Voskuyl AE, Huizinga TW, van de Stadt RJ, Dijkmans BA \& van der Linden S. Influence of glucocorticoids and disease activity on total and high density lipoprotein cholesterol in patients with rheumatoid arthritis. Annals of the Rheumatic Diseases 200362 842-845. (doi:10.1136/ard.62.9.842)

39 Akiyama M, Mawatari T, Nakashima Y, Miyahara H, Yamada H, Okazaki K, Fukushi J, Kondo M, Kishimoto J, Hashimura C et al. Prevalence of dyslipidemia in Japanese patients with rheumatoid arthritis and effects of atorvastatin treatment. Clinical Rheumatology 201534 1867-1875. (doi:10.1007/s10067-015-3049-0)

40 DeFronzo RA \& Goodman AM. Efficacy of metformin in patients with non-insulin-dependent diabetes mellitus. The Multicenter Metformin Study Group. New England Journal of Medicine 1995333 541-549. (doi:10.1056/NEJM199508313330902)

41 Dunn CJ \& Peters DH. Metformin. A review of its pharmacological properties and therapeutic use in non-insulin-dependent diabetes mellitus. Drugs 199549 721-749. (doi:10.2165/00003495-19954905000007)

42 Hermann LS, Schersten B, Bitzen PO, Kjellstrom T, Lindgarde F \& Melander A. Therapeutic comparison of metformin and sulfonylurea, alone and in various combinations. A double-blind controlled study. Diabetes Care 199417 1100-1109. (doi:10.2337/ diacare.17.10.1100)

43 Mayo-Smith W, Hayes CW, Biller BM, Klibanski A, Rosenthal H \& Rosenthal DI. Body fat distribution measured with CT: correlations in healthy subjects, patients with anorexia nervosa, and patients with Cushing syndrome. Radiology 1989170 515-518. (doi:10.1148/ radiology.170.2.2911678)

44 Terzolo M, Pia A, Ali A, Osella G, Reimondo G, Bovio S, Daffara F, Procopio M, Paccotti P, Borretta G et al. Adrenal incidentaloma: a new cause of the metabolic syndrome? Journal of Clinical Endocrinology and Metabolism 200287 998-1003. (doi:10.1210/jcem.87.3.8277)

45 Knowler WC, Barrett-Connor E, Fowler SE, Hamman RF, Lachin JM, Walker EA, Nathan DM \& Diabetes Prevention Program Research G. Reduction in the incidence of type 2 diabetes with lifestyle intervention or metformin. New England Journal of Medicine 2002346 393-403. (doi:10.1056/NEJMoa012512)

46 Bailey CJ \& Turner RC. Metformin. New England Journal of Medicine 1996334 574-579. (doi:10.1056/NEJM199602293340906)

Received 3 August 2016

Revised version received 2 December 2016

Accepted 10 January 2017 